\title{
Spatial Qualifier
}

National Cancer Institute

\section{Source}

National Cancer Institute. Spatial Qualifier. NCI Thesaurus. Code C73706.

A term used to indicate an object's position in space or it's relationship in space to another object. 